\title{
Technology-Enhanced Upper Limb Physical Rehabilitation in Hemiplegic Cerebral Palsy
}

\author{
Josip Glavić*, Stela Rutović, Nikolina Kristić-Cvitanović, Petra Burić and Ana Petrović
}

Polyclinic Glavić, Dubrovnik, Croatia

\begin{abstract}
Cerebral palsy $(\mathrm{CP})$ is the most common lifelong disability affecting motor development in children. Hemiplegic $\mathrm{CP}$ is the most common syndrome in children born at term. Numerous rehabilitation approaches have been reported in children with CP. Recent studies have shown that robot-assisted training can complement conventional therapies in children with cerebral palsy. In this study we present a case of an 18 year old girl with spastic hemiparesis as a form of cerebral palsy, who showed significant recovery after intensive technology-enhanced physical rehabilitation using Armeo spring system.
\end{abstract}

Keywords: Hemiplegic cerebral palsy; Upper limb rehabilitation; Armeo spring

\section{Introduction}

Cerebral palsy $(\mathrm{CP})$ is a neurodevelopmental disorder characterized by movement and posture abnormalities. Incidence of $\mathrm{CP}$ in countries of the Western world is approximately $2-3$ per 1,000 births. Children with CP usually show signs of muscle weakness, sensory deficits, as well as spasticity [1]. Spastic CP is classified in unilateral and bilateral forms [2]. A number of rehabilitation approaches have been reported in children with CP. Bobath concept is a form of conventional therapy, based on motor learning strategies [1]. Recent studies have shown that robot-assisted training can complement conventional therapies in children with cerebral palsy [2,3]. In this study we present a case of an 18 year old girl with spastic hemiparesis as a form of cerebral palsy, who showed remarkable recovery after intensive technology-enhanced physical rehabilitation using Armeo spring system (Figure 1).

\section{Materials and Methods}

An 18 year old girl with a diagnosis of a cerebral palsy with a rightsided spastic hemiparesis came to our clinic for rehabilitation treatment using Armeo Spring system. She was a prematurely born child and her previous medical history included Achill tendon operation at the age of three. Since her birth and before coming to our clinic she received standard physical therapy using Bobath treatment, administered by a physiotherapist, for 1 hour per day, 5 days a week. The locomotor system was assessed at the beginning and end of the treatment programme with Armeo Spring. Main outcome measurements included FuglMayer (FM) score as a measure for motor assessment of the upper extremity, Functional Independence Measure (FIM) as a parameter for global functional evaluation and Modified Ashworth Scale (MAS) as a measure of muscle spasticity [4]. Training frequency was 5 times per week for 12 weeks. One session lasted $40 \mathrm{~min}$.

\section{Results}

On her initial physical examination right shoulder abduction and anteflexion were terminally limited. Muscle strength in elbow flexors and extensors was 4/5. Right fist was held in wrist flexion, she could not actively perform right wrist extension and cold not completely clench her fist. Grasp was crude and release was slow, she could hold objects for manipulation by her left hand. She could not perform thumb opposition. MMT (manual muscle test) of right fist was $3 / 5$. Right forearm pronation was in the normal range, but supination could only be initiated. Left leg muscle strength was $4 / 5$. Muscle tone on right extremities was increased. Baseline FM score was 42, FIM 116 and MAS was 2.
After treatment with the arm weight support device hand function improved. Right shoulder abduction and anteflexion were in the normal range. Muscle strength in elbow flexors and extensors was 5/5. Right fist was held in wrist flexion, she could not actively perform right wrist extension, but there was an increase in muscle strength and range of movements. Grasp, release and opposition activities improved. MMT of right fist was 4/5. Right forearm pronation was in the normal range, and supination improved. There was some reduction in muscle spasticity. FM score increased significantly to 50, FIM increased to 120 and MAS decreased to $1+$.

The results of treatment with Armeo Spring showed significant improvement measured in FM score and FIM comparing to treatment with conventional physical therapy by Bobath method. So far a number of rehabilitation approaches have been reported in children

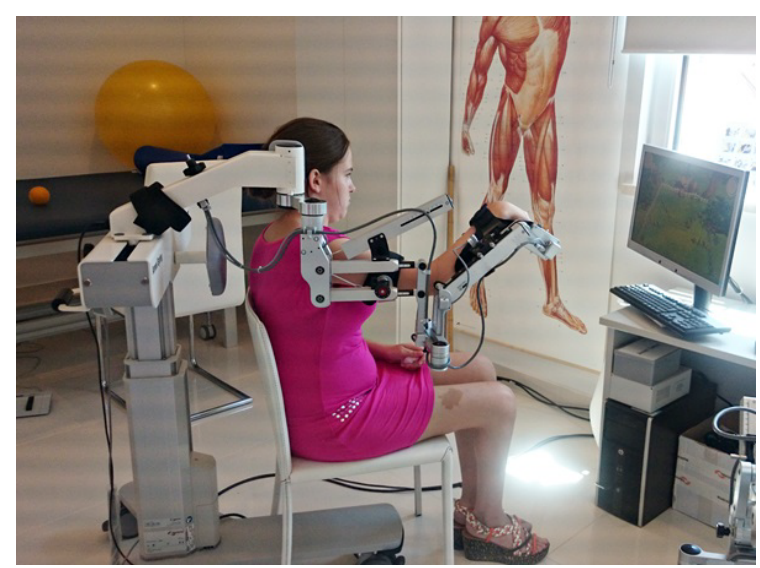

Figure 1: Showing rehabilitation treatment with Armeo Spring system.

*Corresponding author: Josip Glavić, MD, Polyclinic Glavić, Dubrovnik, Croatia, Tel: 0038520435565; Fax: 0038520438387; E-mail: josko@poliklinika-glavic.hr

Received August 09, 2016; Accepted August 29, 2016; Published August 31, 2016

Citation: Glavić J, Rutović S, Cvitanović NK, Burić P, Petrović A (2016) TechnologyEnhanced Upper Limb Physical Rehabilitation in Hemiplegic Cerebral Palsy. Int J Neurorehabilitation 3: 225. doi: 10.4172/2376-0281.1000225

Copyright: $\odot 2016$ Glavić J, et al. This is an open-access article distributed under the terms of the Creative Commons Attribution License, which permits unrestricted use, distribution, and reproduction in any medium, provided the original author and source are credited. 
with CP such as anticonvulsants, bimanual training, botulinum toxin, bisphosphonates, casting, constraint-induced movement therapy, context-focused therapy, diazepam, fitness training, goal-directed training, hip surveillance, home programmes, occupational therapy after botulinum toxin, pressure care, selective dorsal rhizotomy, and robot assisted training [5]. The Bobath technique is the most common method of motor stimulation and it is used worldwide. It is designed to promote practice of task- specific, functional skills by active participation. Its aims are to inhibit spasticity and abnormal patterns of movement, improve postural alignment, and to facilitate normal automatic and voluntary movements [1]. Although it improves motor function, recent review study has shown that the results of treatment children with CP using Bobath therapy have limited effectiveness. The main disadvantages of Bobath therapy stated in this study are not significantly improving muscle contracture and tone, since it aims to reduce hyper-reflexia by repositioning the limb, thereby providing local effects, and not working on centrally driven spasticity long term [5]. A recent meta- analysis also showed weak to moderate effects of Bobath therapy on improving quality of upper limb movement and fine motor skills despite its common use in clinical practice [6].

Children often do not find this kind of therapy motivating, and do not achieve adequate training duration and intensity, which potentially leads to reduced therapy effects.

Armeo Spring is a system used for upper limb rehabilitation. It is an exoskeleton which provides gravity support for the affected upper limb by means of a spring mechanism and magnifies any residual active movement of the hemiparetic arm. As a seven degree of freedom orthosis with built-in position sensors it gives information about specific movement parameters (resistance, strength, range of motion and coordination), with a possibility of system sensitivity adjustment depending on the patient's condition. In its distal region it includes a grasp pressure sensitive handgrip which allows a graded performance of grasp and release exercises. This system enables participants to practice independent task-oriented and repetitive movements in a virtual three- dimensional learning environment, involving central neural pathways related to proprioceptive and visual feedback processing $[2,4,7]$.

This kind of interactive approach is very motivating for patient and allows tasks to be executed repeatedly with higher intensity.

This is a relatively new technology, so far two studies have described favorable results in upper limb rehabilitation using Armeo Spring in children with CP $[2,3]$, and further studies are required. Our study has also shown significant recovery in a relatively short time period using patient motivating and interesting intensive task- specific training.

In conclusion, our results showed that Armeo Spring system is a useful method for improving upper limb functionality. It is advisable to implement its use additionally to conventional therapy so that children with CP could achieve the best possible outcomes.

\section{References}

1. Mayston MJ (2001) People with cerebral palsy: effects of and perspectives for therapy. Neural Plast 8: 51-69.

2. Turconi AC, Biffi E, Maghini C, Peri E, Servodio IF, et al. (2015) May new technologies improve upper limb performance in grown up diplegic children? Eur J Phys Rehabil Med.

3. Peri E, Biffi E, Maghini C, Servodio IF, Gagliardi C, et al. (2016) Quantitative evaluation of performance during robot-assisted treatment. Methods Inf Med 55: 84-88.

4. Bartolo M, De Nunzio AM, Sebastiano F, Spicciato F, Tortola P, et al. (2014) Arm weight support training improves functional motor outcome and movement smoothness after stroke. Funct Neurol 29: 15-21.

5. Novak I, Mclntyre S, Morgan C, Campbell L, Dark L, et al. (2013) A systematic review of interventions for children with cerebral palsy: State of the evidence. Dev Med Child Neurol 55: 885-910.

6. Sakzewski L, Ziviani J, Boyd RN (2014) Efficacy of upper limb therapies for unilateral cerebral palsy: A meta-analysis. 133: e175-e204.

7. Colomer C, Baldovi A, Torrome S, Navarro MD, Moliner B, et al. (2013) Efficacy of Armeo ${ }^{\circledR}$ Spring during the chronic phase of stroke. Study in mild to moderate cases of hemiparesis. Neurologia 28: 261-267. 